\section{Nachhaltiges Sanieren im Bestand}

\author{
In den kommenden Jahren muss ein großer Wohnungsbestand aus den $\mathbf{5 0}$-er und \\ 60-er Jahren saniert werden. Dies ist eine große Herausforderung für die Woh- \\ nungswirtschaft in Deutschland. Nachhaltiges Sanieren ist hierfür ein ökolo- \\ gisch, ökonomisch und sozial tragfähiger Weg. Die Entwicklung geeigneter Kon- \\ zepte stellt sowohl an die Unternehmen als auch die transdisziplinäre Forschung \\ erhebliche Anforderungen.
}

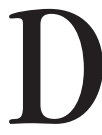

Von Kathrin Ankele as Konzept der nachhaltigen Sanierung bietet die Chance, langfristige soziale Stabilität der Wohnsiedlungen, Umweltverträglichkeit und wirtschaftliche Tragfähigkeit zu verbinden. Darüber hinaus beinhaltet die verstärkte Orientierung auf den Bestand erhebliche Nachhaltigkeitspotenziale allein schon durch den deutlich geringeren Flächen- und Ressourcenvebrauch gegenüber dem Neubau. Ein ökoforums-Kooperationsprojekt hat diese Potenziale zusammen mit dem Praxispartner Nassauische Heimstätte näher untersucht und wesentliche Ergebnisse in einem Leitfaden zusammengefasst (1).

Die Ziele des Vorhabens umfassten folgende Bereiche:

- Entwicklung eines Konzepts zur nachhaltigen Bestandssanierung, das ökologische, soziale und wirtschaftliche Zielsetzungen zu Maßnahmenbündeln integriert,

praktische Erprobung der Maßnahmenbündel durch Umsetzung in Modellsiedlungen,

- Schaffung unternehmensinterner Voraussetzungen für nachhaltiges Sanieren und

- Sicherstellung der Übertragbarkeit der Projektergebnisse in Bezug auf die Vorgehensweise und die entwickelten Maßnahmenbündel.

Die Nassauische Heimstätte verfügt im RheinMain-Gebiet über ca. 45.000 Wohnungen. Aus diesem Wohnungsbestand wurden die HansBöckler-Siedlung in Offenbach und zwei Siedlungen in Bad-Soden (Taunus) als Modellsiedlungen für das Vorhaben ausgewählt.

Ausgangspunkt der Maßnahmenentwicklung war eine Bestandsaufnahme, die den baulich-technischen Sanierungsbedarf, die Wünsche und Anforderungen der MieterInnen, den Handlungsbedarf im Hinblick auf soziale Stabilisierung der Siedlungen, Verbesserungspotenziale im Sanierungsprozess des Wohnungsunternehmens und schließlich dessen Kooperationsbeziehungen zu Kommunen und Handwerksunternehmen beleuchtete (2). Diese projektspezifischen Voraussetzungen wurden den ökologischen, sozialen und ökonomischen Zielen gegenüber gestellt, die die Enquete-Kommission für das Handlungsfeld Bauen und Wohnen entwickelte (3). Darüber hinaus wurden Entwicklungstendenzen bezüglich der Mieterschaft und der ökonomischen Rahmenbedingungen im RheinMain-Gebiet einbezogen.

In einem iterativen Prozess, der im Projektverlauf immer stärker von den unternehmensinternen Projektteams getragen wurde, wurden die Maßnahmenbündel zunehmend konkretisiert. Am Ende des Forschungsvorhabens war die Planung der ersten Bauabschnitte abgeschlossen und in einer der Modellsiedlungen wurde mit der Modernisierung begonnen. Alle Baumaßnahmen bei den insgesamt rund 800 Wohnungen werden voraussichtlich in drei Jahren fertiggestellt sein. Das Vorhaben zeichnet sich somit durch einen sehr hohen Umsetzungsgrad aus.

\section{Ausgewählte Maßnahmen}

Kernbestandteil der baulich-technischen Maßnahmen ist die ökologische Gebäudesanierung. Sie umfasst unter anderem Maßnahmen zur Wärmedämmung der Außenhülle, moderne Elektroinstallationen und Sanitäreinrichtungen sowie die Fenstererneuerung. Daneben wurden ein nachhaltiges Abfallkonzept und Maßnahmen zum Rückbau und künftigen Verzicht auf schadstoffhaltige Baustoffe entwickelt .

Im Konzept der nachhaltigen Sanierung stehen die baulich-technischen Maßnahmen jedoch nicht isoliert. Sie werden verknüpft mit sozialen Maßnahmen, um gemeinsam die Mieterzufriedenheit und die Attraktivität der Siedlungen zu erhöhen. Die Verzahnung wird an Hand der Schaffung von Gemeinschaftsräumen deutlich, die auf der Grundlage baulicher Maßnahmen einen verstärken Austausch zwischen den MieterInnen und eine Belebung der Nachbarschaften ermöglichen. Auch die Nachrüstung von Erdgeschosswohnungen für weniger mobile MieterInnen sowie die Grundrissumgestaltung zur Flexibilisierung der Wohnungsnutzung im Hinblick auf Änderungen der MieterInnenstruktur - sind Beispiele hierfür.

Darüber hinaus wurden sanierungsbegleitende Unterstïtzungsmaßnahmen durch SanierungshelferInnen entwickelt, deren Stellen im Rahmen von Beschäftigungsförderung geschaffen wurden. Dazu zählen die Unterstützung der Wohnungsverwaltung ebenso wie Leistungen für die MieterInnen, beispielsweise Beratung und Umzugsmanagement.

Unverzichtbar für nachhaltiges Sanieren ist die aktive Einbeziehung der MieterInnen. Dazu zählt eine frühzeitige Information über die geplanten Baumaßnahmen, kontinuierliche Informationen über den Baufortschritt und Ansprechpartner vor Ort. Außerdem sollten Gestaltungsspielräume der MieterInnen offen gelegt werden, etwa bei Fliesen- und Bodenfarbe, und auf Mieterversammlungen oder einer Planungswerkstatt über die Umgestaltung der Außenanlagen diskutiert werden.

\section{Unternehmensinterne Voraussetzungen}

Um nachhaltiges Sanieren umsetzen zu können, müssen Voraussetzungen im Wohnungsunternehmen geschaffen werden. Neben einem nachhaltigen Bestandsmanagement, das die Auswahl der Siedlungen und der durchzuführenden Maßnahmen beinhaltet, ist die abteilungsübergreifende Zusammenarbeit bei der Sanierungsplanung und -vorbereitung zentral. Insbesondere soll sichergestellt werden, dass die sozialen Aspekte ausreichend in den Planungsprozess integriert werden. Hierfür wurden Projektteams aus den dezentralen Wohnungsverwaltungen, den technischen Abteilungen, dem Sozialmanagement, der Projektsteuerung und fallweise weiteren Fachkräften gebildet. Deren Aufgabe bestand darin, die Maßnahmen zur nachhaltigen Sanierung der beiden ausgewählten Modellsiedlungen bis zur Verabschiedungsreife durch die Geschäftsleitung vorzubereiten. Im Vorhaben konnten die Projektteams auf Maßnahmenvorschläge der Institute zurückgreifen und diese weiterentwickeln. Diese bislang ungewohnte Arbeitsweise in Projektteams unter Leitung der Wohnungsverwaltungen wurde durch Arbeitspläne und Meilensteine strukturiert. 
Die Steuerung einer Komplettmodernisierung ist ein komplexes Vorhaben, das durch verschiedene Instrumente unterstïtzt werden kann. Im Vorhaben wurde ein „Handbuch der nachhaltigen Komplettsanierung" entwickelt, das die Abläufe strukturiert und systematisiert und alle relevanten Informationen und Unterlagen zusammenfasst (4). $\mathrm{Zu}$ jeder Zeit können der Projektfortschritt und die weiteren Schritte dargestellt werden. Auch die Zusammenarbeit der Projektteams wird durch dieses Dokument erleichtert, da dezentral darauf zurïckgegriffen werden kann.

\section{Qualifizierung der Handwerker}

Sanierungsmaßnahmen stellen immer eine Belästigung für die MieterInnen dar. Um diese so gering wie möglich zu halten, können verschiedene Maßnahmen ergriffen werden. Zentral ist eine möglichst kurze Zeitspanne der Arbeiten, die durch optimale Abstimmung der Einzelgewerke erreicht werden kann. Im Vorhaben wurde diskutiert, inwiefern eingespielte Handwerkerkooperationen zu diesem Ziel beitragen können. Ausgewählte Handwerkerkooperationen werden bei der Ausschreibung der Bauvorhaben einbezogen, um deren „Wettbewerbsfähigkeit“ gegenüber Einzelanbietern zu testen.

In jedem Fall können geschulte Sanierungshandwerker zu dieser Zielsetzung beitragen. Im Vorhaben wurde ein Curriculum Bewohnerfreundliches Sanieren entwickelt, das speziell auf die Erweiterung der sozialen und kommunikativen Fähigkeiten der Handwerker abzielt. Die Qualifizierungsmaß- nahme soll nun mit Mitteln des Landes Hessen und der EU umgesetzt werden, wobei die Nassauische Heimstätte als ein Träger fungiert.

\section{- Nachbemerkung zu inter- und transdisziplinärer Forschung}

Inter- und transdisziplinäre Forschungsvorhaben sind derzeit sehr modern. Das Bundesforschungsministerium (BMBF) und viele andere Fördermittelgeber legen zunehmend Wert auf die Zusammenarbeit verschiedener Disziplinen und die intensive Beteiligung von Praxispartnern. Das Forschungsvorhaben kann als erfolgreiches Beispiel hierfür gewertet werden. In einer konzeptionellen Studie könnten möglicherweise weiter- gehende Vorschläge entwickelt werden. Der unbestrittene Erfolg des Vorhabens liegt demgegenüber darin, dass die Ergebnisse in einem großen Wohnungsbestand umgesetzt werden und dieses Nachhaltigkeitspotenzial tatsächlich erschlossen wird. Voraussetzung hierfür waren sehr engagierte MitarbeiterInnen seitens des Praxispartners sowie ein intensiver und zeitaufwändiger Abstimmungsprozess.

Inter- und transdisziplinäre Forschung ist kein Selbstzweck und auch nicht ohne Zusatzaufwand umzusetzen. Es wäre wünschenswert, diesbezügliche Erfahrungen, die beispielsweise in den aktuellen BMBF-Förderprogrammen gesammelt werden, zusammenzutragen und zu diskutieren. Aus Erfolgs- und Misserfolgsbeispielen sollten Forschungsnehmer und Fördermittelgeber lernen. Ziel wäre unter anderem, ein differenziertes
Bild darüber zu erhalten, wann inter- und transdisziplinäre Ansätze sinnvoll sind und welche Voraussetzungen hierfür geschaffen werden müssen.

\section{Anmerkungen}

(1) Projektverbund Nachhaltiges Sanieren im Bestand (Hrsg.): Nachhaltiges Sanieren im Bestand. Leiffaden für die Wohnungswirtschaft. Berlin, Darmstadt, Frankfurt/ Main, Freiburg 2001. Internet-Download: www.isoe.de/ ftp/nasaleit.pdf. Das vom BMBF geförderte Forschungsvorhaben "Nachhaltiges Sanieren im Bestand - integrierte Dienstleistungen für zukunftsfähige Wohnstile" wurde im Frühjahr dieses Jahres abgeschlossen. Bearbeitet wurde es von ISOE (Projektleitung), Öko-Institut und IÖW in Kooperation mit der nhgip (Nassauische Heimstätte - Gesellschaft für innovative Projekte im Wohnungsbau).

(2) Vgl. dazu ausführlicher Stieß, I.: Frischer Wind in alten Mauern. In: Politische Ökologie Nr. 71: NeuHausen - nachhaltiges Bauen und Wohnen.

(3) Deutscher Bundestag (Hrsg.): Konzept Nachhaltigkeit Vom Leitbild zur Umsetzung. Abschlussbericht der EnqueteKommission "Schutz des Menschen und der Umwelt - Ziele und Rahmenbedingungen einer nachhaltig zukunftsverträglichen Entwicklung" des 13. Deutschen Bundestages, Bonn 1998.

(4) Das Handbuch ist im bislang noch unveröffentlichten Endbericht dokumentiert

\section{Die Autorin}

Kathrin Ankele leitet das Forschungsfeld Ökologische Unternehmenspolitik im IÖW.

Kontakt: IÖW, Potsdamer Str. 105, 10785 Berlin.

Tel. 030/ 884594-21, Fax 030/ 8825439,

E-mail: Kathrin.Ankele@ioew.de

\section{Unternehmerische}

\section{Nachhaltigkeit umsetzen}

\section{Die Balanced Scorecard ist ein modernes Instrument zur operativen Umsetzung strategischer Ziele. Wie kann sie praxisnah weiterentwickelt werden, um unter- nehmerische Nachhaltigkeit in das allgemeine Managementsystem zu integrieren? Hierzu sind verschiedene Wege möglich. Erste konzeptionelle Ansätze wurden an- hand eines Fallbeispiels im Rahmen des VöW-Workshops "Die Politik allein wird es nicht richten?!” am 12. Mai 2001 in Berlin erprobt und diskutiert.}

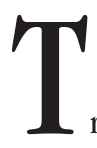

Von T. Bieker, C. U. Gminder, T. Hahn und $M$. Wagner merische Nachhaltigkeit immer noch ein unklares Konstrukt: Unternehmen glänzen wohl mit voll- mundigen Umwelt- und neuerdings Nachhaltigkeits-Politiken, unterzeichnet von höchster Ebene. Bei der Umsetzung hapert es jedoch: Umweltmanagementsysteme sind wenig effektiv, zu operativ ausgerichtet und sind selten in die allgemeinen
Managementsysteme integriert (1). Kann der Einbezug von Umwelt und Sozialem in die Balanced Scorecard, dem „Shooting-Star“ der Managementsysteme, diese Schwierigkeiten lösen (2)? Dieser Frage gehen zurzeit zwei Forscherteams der Universitäten Lüneburg und Sankt Gallen im Rahmen eines BMBF-Projektes nach (3). Ausgehend von der Kritik an traditionellen Controlling- und Steuerungssystemen wurde Anfang der 90-er Jahre die Balanced Scorecard (BSC) vor allem zur Schließung der Lücke zwischen strategischer Planung und operativer Umsetzung entwikkelt (4). Sie zielt auf das Umsetzen von Strategien in die operative Ebene und besteht aus einer Scorecard mit folgenden Elementen (siehe Abb. 1):

- Vier Perspektiven anhand derer externe Anspruchsgruppen (Anteilseigner, Kunden) und interne Aspekte (Prozesse, Lernen) berücksichtigt werden; 
(c) 20I0 Authors; licensee IÖW and oekom verlag. This is an article distributed under the terms of the Creative Commons Attribution Non-Commercial No Derivates License (http://creativecommons.org/licenses/by-nc-nd/3.o/), which permits unrestricted use, distribution, and reproduction in any medium, provided the original work is properly cited. 